The Journal of Nonlinear $\mathbf{S}$ ciences and Applications

http://www.tjnsa.com

\title{
EXISTENCE AND UNIQUENESS OF FRACTIONAL DIFFERENTIAL EQUATIONS WITH INTEGRAL BOUNDARY CONDITIONS
}

\author{
TAIGE WANG ${ }^{1}$ AND FENG XIE ${ }^{2 *}$
}

\begin{abstract}
In this article, the recently developed monotonous iterative method is used to investigate fractional differential equations involving Riemann-Liouville differential operators with integral boundary conditions. The existence and uniqueness of solutions are obtained.
\end{abstract}

\section{INTRODUCTION}

We consider the following fractional differential problem with integral boundary condition

$$
\begin{aligned}
& D^{q} x(t)=f(t, x), \quad t \in J=[0, T], \quad T \geq 0, \\
& x(0)=\lambda \int_{0}^{T} x(s) d s+d, \quad d \in \mathbb{R} .
\end{aligned}
$$

where $0<q<1, \lambda$ is 1 or -1 and $f \in C[J \times \mathbb{R}, \mathbb{R}]$. $D^{q}$ denotes the fractional derivative of order $q$ in the sense of Riemann-Liouville. Problem (1.1) with $q=1$ was investigated by Jankowski [5]. Very recently, the basic theory of problem (1.1) with $\lambda=0$ has been obtained by Lakshmikantham and Vatsala in a series of work [6, 7, 8]. The monotonous iterative method for fractional differential equations and the theory of fractional differential equalities have also been developed.

The significance of fractional differential equations has been displayed in the research of applied mathematics these years, especially in the study on disordered semiconductors and viscoelastic materials; see [1, 2, 13, 14, for instance. Because most of nonlinear fractional differential equations do not exact analytic solutions, various analytic approximation and numerical methods have been proposed and

Date: Received: 2 April 2008; Revised: 15 Dec 2008.

* Corresponding author.

2000 Mathematics Subject Classification. Primary 26A33; Secondary 45D05.

Key words and phrases. Fractional differential equations, integral boundary condition, monotonous iterative method. 
developed recently. For example, the He's homotopy perturbation method $[9,10$, [12, 15] and variational iteration method [3, 4, 11] have been successfully applied to solve a variety of nonlinear fractional differential equations.

In the present article, we shall discuss the existence and uniqueness of problem (1.1) by employing the monotonous iterative method recently developed by Lakshmikantham and Vatsala. Note that problem (1.1) is equivalent to the following integral equation:

$$
x(t)=\lambda \int_{0}^{T} x(s) d s+d+\frac{1}{\Gamma(q)} \int_{0}^{t}(t-s)^{q-1} f(s) d s, \quad 0 \leq t \leq T,
$$

where $\Gamma(q)$ is the Gamma Function of $q$.

The layout of this paper is as follows. In Section 2, we employ the monotonous iterative method to testify the existence and uniqueness of solution to problem (1.1) in the case of $\lambda=1$. The similar way to establish the corresponding theory of solution to problem (1.1) when $\lambda=-1$ is given in Section 3 .

\section{CASE $\lambda=1$}

Before the detailed establishment, let us introduce some definitions and assumptions.

Definition 2.1. Assume that there exist $v_{0}(t)$ and $w_{0}(t)$ which are locally Hölder continuous and satisfy:

$$
\begin{gathered}
D^{q} v_{0}(t) \leq f\left(t, v_{0}(t)\right), \quad v_{0}(0) \leq \int_{0}^{T} v_{0}(s) d s, \\
D^{q} w_{0}(t) \geq f\left(t, w_{0}(t)\right), \quad w_{0}(0) \geq \int_{0}^{T} w_{0}(s) d s .
\end{gathered}
$$

Then we call $v_{0}(t)$ and $w_{0}(t)$ lower and upper solutions of problem (1.1), respectively.

We make the following assumptions:

$\left(H_{1}\right) f \in C(J \times \mathbb{R}, \mathbb{R})$;

$\left(H_{2}\right) v_{0}(t), w_{0}(t) \in C^{1}(J, \mathbb{R})$ are lower and upper solutions of problem (1.1);

$\left(H_{3}\right)$ There exists $0 \leq M \leq \frac{1}{T^{q} \Gamma(1-q)}$ such that $f(t, x)-f(t, y) \geq-M(x-y)$ for $x \geq y$.

Lemma 2.2. If $H_{1}$ and $H_{2}$ hold, and there exists $L \in\left(0, \frac{1}{T^{q} \Gamma(1-q)}\right)$ such that $f(t, x)-f(t, y) \leq L(x-y)$ for $y \leq x$. Then, $v(0) \leq w(0)$ implies $v(t) \leq w(t)$.

Actually, the proof in the case of integral boundary condition is the same as that in the initial value condition because the integral condition has the same definition like the initial one used in the proofs. And the proof of the latter can be found in [8], so we omit it here. 
Lemma 2.3. If $\left(H_{1}\right),\left(H_{2}\right)$ and $\left(H_{3}\right)$ hold, and the mapping $A: A v_{0}=v_{1}, A w_{0}=$ $w_{1}$ is defined as:

$$
\begin{array}{rr}
D^{q} v_{1}=f\left(t, v_{0}\right)-M\left(v_{1}(t)-v_{0}(t)\right), & v_{1}(0)=\int_{0}^{T} v_{0}(s) d s+d, \\
D^{q} w_{1}=f\left(t, w_{0}\right)-M\left(w_{1}(t)-w_{0}(t)\right), & w_{1}(0)=\int_{0}^{T} w_{0}(s) d s+d .
\end{array}
$$

Then the mapping A has the properties:

(a) $A v_{0} \geq v_{1}, A w_{0} \leq w_{1}$;

(b) on $\left[v_{0}, w_{0}\right]=\left[x \in C(J, \mathbb{R}): v_{0} \leq w_{0}\right]$, $A$ is a monotonous operator.

Proof. (a). Note that the right side of equations in (2.1) meets Lipshitz condition, which warrants the uniqueness of the solution $v_{1}, w_{1}$.

Setting $p=v_{1}-v_{0}$, we have

$$
\begin{aligned}
D^{q} p(t) & =f\left(t, v_{0}(t)\right)-M\left[v_{1}(t)-v_{0}(t)\right]-f\left(t, v_{0}(t)\right) \\
& \geq-M\left[v_{1}(t)-v_{0}(t)\right] \\
& =-M p(t) \\
p(0) & =v_{1}(0)-v_{0}(0) \geq 0 .
\end{aligned}
$$

Note that the initial value problem $D^{q} x(t)=0, x(0)=x_{0}$ has a unique solution $x(t) \equiv 0$ on $J$. It follows from Lemma 2.2 that $p(t) \geq 0$, which implies $v_{1}(t) \geq$ $v_{0}(t)$. Similarly, we can get $w_{1}(t) \leq w_{0}(t)$.

(b). For $\sigma_{1}, \sigma_{2} \in\left[v_{0}, w_{0}\right], \sigma_{2} \geq \sigma_{1}$, setting $p=A \sigma_{2}-A \sigma_{1}=x_{2}-x_{1}$, we have

$$
\begin{aligned}
D^{q} p & =f\left(t, \sigma_{2}(t)\right)-M\left(x_{2}-\sigma_{2}\right)-f\left(t, \sigma_{1}(t)\right)+M\left(x_{1}-\sigma_{1}\right) \\
& \geq-M\left(\sigma_{2}-\sigma_{1}\right)+M\left(\sigma_{2}-\sigma_{1}\right)-M\left(x_{2}-x_{1}\right) \\
& =-M\left(x_{2}-x_{1}\right) \\
& =-M p(t), \\
p(0) & =x_{2}(0)-s_{1}(0) \\
& =\int_{0}^{T}\left[\sigma_{2}(s)-\sigma_{1}(s)\right] d s \\
& \geq 0 .
\end{aligned}
$$

It follows from Lemma 2.2 that $A \sigma_{2} \geq A \sigma_{1}$, which implies that $A$ is a monotonous operator. The proof of Lemma 2.3 is completed.

Theorem 2.4. Assume that $H_{1}, H_{2}$ and $H_{3}$ hold, and the mapping $A: A v_{n}=$ $v_{n+1}, A w_{n}=w_{n+1}$ are defined as:

$$
\begin{gathered}
D^{q} v_{n+1}=f\left(t, v_{n}\right)-M\left(v_{n+1}(t)-v_{n}(t)\right), \quad v_{n+1}(0)=\int_{0}^{T} v_{n}(s) d s+d, \\
D^{q} w_{n+1}=f\left(t, w_{n}\right)-M\left(w_{n+1}(t)-w_{n}(t)\right), \quad w_{n+1}(0)=\int_{0}^{T} w_{n}(s) d s+d .
\end{gathered}
$$

Then there exist monotone sequences $\left\{v_{n}\right\},\left\{w_{n}\right\}$ such that $v_{n} \rightarrow v(t), w_{n} \rightarrow w(t)$ as $n \rightarrow \infty$, where $(v, w)$ are the extremal solutions of (1.1) on $0 \leq t \leq T$. 
Proof. The definition of the mapping $A$ gives $A v_{n-1}=v_{n}, A w_{n-1}=w_{n}$. From (a) and (b) in Lemma 2.3, it is not difficult to get:

$$
v_{0} \leq v_{1} \leq \ldots \leq v_{n-1} \leq v_{n} \leq w_{n} \leq w_{n-1} \leq \ldots \leq w_{2} \leq w_{1} \leq w_{0}, t \in J .
$$

Apparently, there exist $v, w$ such that $\lim _{n \rightarrow \infty} v_{n}(t)=v(t), \lim _{n \rightarrow \infty} w_{n}(t)=w(t)$. And $v, w$ are solutions of problem (1.1). Next we will prove that $v, w$ are the minimal and maximal solutions of problem (1.1), respectively.

Let $x(t) \in\left[v_{0}, w_{0}\right]$ is a solution of (1.1) different from $v(t)$ and $w(t)$, so there exist $k \in N$ such that $v_{k}(t) \leq x(t) \leq w_{k}(t)$. Setting $p(t)=x-v_{k+1}$, we have

$$
\begin{aligned}
D^{q} p(t) & =f(t, x(t))-f\left(t, v_{k}(t)\right)+M\left[v_{k+1}(t)-v_{k}(t)\right] \\
& \geq-M\left[x(t)-v_{k}(t)\right]+M\left[v_{k+1}(t)-v_{k}(t)\right] \\
& =M\left[v_{k+1}(t)-x(t)\right] \\
& =M p(t), \\
p(0) & =x(0)-v_{k+1}(0) \\
& =\int_{0}^{T}\left[x(s)-v_{k}(s)\right] d s \\
& \geq 0 .
\end{aligned}
$$

By Lemma 2.2, we get that $p(t)=x(t)-v_{k+1}(t) \geq 0$, which implies $x(t) \geq v(t)$ by letting $k \rightarrow \infty$. For the same sake, we can also get $x(t) \leq w(t)$. Now we can see that $v, w$ are the minimal solution and the maximal solution to (1.1).

Theorem 2.5. Assume that $H_{1}, H_{2}$ and $H_{3}$ hold, and there exists $L \in\left(0, \frac{1}{T^{q} \Gamma(1-q)}\right)$ such that $f(t, x)-f(t, y) \leq L(x-y)$ for $y \leq x$. Suppose further that $\lim _{n \rightarrow \infty} \| w_{n}-$ $v_{n} \|=0$ where the norm is defined as $\|f\|=\int_{0}^{T}|f(s)| d s$. Then the solution of problem (1.1) is unique.

Proof. Setting $p=w-v$, we have

$$
\begin{aligned}
D^{q} p(t) & =f(t, w(t))-f(t, v(t)) \\
& \leq M[w(t)-v(t)] \\
& =M p(t), \\
p(0) & =w(0)-v(0) \\
& =\lim _{n \rightarrow \infty}\left[w_{n}(0)-v_{n}(0)\right] \\
& =\lim _{n \rightarrow \infty}\left\|w_{n-1}-v_{n-1}\right\| \\
& =0 .
\end{aligned}
$$

Again with Lemma 2.2, we could get $p(t) \leq 0$. As a result, it follows $v(t) \geq w(t)$. But considering $v(t) \geq w(t)$, we have $v(t) \equiv w(t)$.

\section{CASE $\lambda=-1$}

Definition 3.1. Assume that there exist $v_{0}(t)$ and $w_{0}(t)$ which are locally Hölder continuous and satisfy: 


$$
\begin{gathered}
D^{q} v_{0}(t) \leq f\left(t, v_{0}(t)\right), \quad v_{0}(0) \leq-\int_{0}^{T} w_{0}(s) d s \\
D^{q} w_{0}(t) \geq f\left(t, w_{0}(t)\right), \quad w_{0}(0) \geq-\int_{0}^{T} v_{0}(s) d s .
\end{gathered}
$$

Then we call $v_{0}(t)$ and $w_{0}(t)$ the weakly coupled lower and upper solutions of problem (1.1), respectively.

We make assumptions:

$\left(H_{4}\right)$ There exist weakly coupled upper and lower solutions $w_{0}$ and $v_{0}$ of problem (1.1);

$\left(H_{5}\right)$ There exists $M \in\left[0, \frac{1}{T^{q} \Gamma(1-q)}\right]$ such that $M(x-y) \geq f(t, x)-f(t, y) \geq$ $-M(x-y)$ for $x \geq y$;

$\left(H_{6}\right)$ The sequences $\left\{v_{n}\right\}$ and $\left\{w_{n}\right\}$ given by the mapping $A$ are weakly coupled, and $\lim _{n \rightarrow \infty}\left\|w_{n}-v_{n}\right\|=0$ where the norm is defined as $\|f\|=\int_{0}^{T}|f(s)| d s$.

Lemma 3.2. Assume that $\left(H_{4}\right)$ and $\left(H_{5}\right)$ hold, and the mapping $A: A v_{0}=$ $v_{1}, A w_{0}=w_{1}$ is defined as:

$$
\begin{array}{cc}
D^{q} v_{1}=f\left(t, v_{0}\right)-M\left(v_{1}(t)-v_{0}(t)\right), & v_{1}(0)=-\int_{0}^{T} w_{0}(s) d s+d, \\
D^{q} w_{1}=f\left(t, w_{0}\right)-M\left(w_{1}(t)-w_{0}(t)\right), & w_{1}(0)=-\int_{0}^{T} v_{0}(s) d s+d .
\end{array}
$$

Then the conclusions (a) and (b) in Lemma 2.3 hold.

Proof. (a). Note that the right side of equations in (3.1) satisfy Lipshitz condition, which assures the uniqueness of solution to (1.1).

Setting $p=v_{1}-v_{0}$, we have

$$
\begin{aligned}
D^{q} p(t) & =f\left(t, v_{0}(t)\right)-M\left[v_{1}(t)-v_{0}(t)\right]-f\left(t, v_{0}(t)\right) \\
& \geq-M\left[v_{1}(t)-v_{0}(t)\right] \\
& =-M p(t) \\
p(0) & =v_{1}(0)-v_{0}(0) \\
& =-\int_{0}^{T} w_{0}(s) d s+\int_{0}^{T} w_{0}(s) d s \\
& =0
\end{aligned}
$$

It follows from that $p(t) \geq 0$ which implies $v_{1}(t) \geq v_{0}(s)$. For the same sake, $w_{1}(t) \leq w_{0}(t)$. 
(b). For $\sigma_{1}, \sigma_{2} \in\left[v_{0}, w_{0}\right], \sigma_{2} \geq \sigma_{1}$, let $\sigma_{1}^{\star}, \sigma_{2}^{\star}$ are also coupled solutions, and $\sigma_{1}^{\star} \geq \sigma_{2}^{\star}$. Setting $p=A \sigma_{2}-A \sigma_{1}=x_{2}-x_{1}$, we have

$$
\begin{aligned}
D^{q} p & =f\left(t, \sigma_{2}(t)\right)-M\left(x_{2}-\sigma_{2}\right)-f\left(t, \sigma_{1}(t)\right)+M\left(x_{1}-\sigma_{1}\right) \\
& \geq-M\left(\sigma_{2}-\sigma_{1}\right)+M\left(\sigma_{2}-\sigma_{1}\right)-M\left(x_{2}-x_{1}\right)=-M\left(x_{2}-x_{1}\right) \\
& =-M p(t), \\
p(0) & =x_{2}(0)-x_{1}(0) \\
& =\int_{0}^{T}\left[-\sigma_{2}^{\star}(s)+\sigma_{1}^{\star}(s)\right] d s \\
& \geq 0 .
\end{aligned}
$$

It follows from Lemma 2.2 that $x_{2}(t) \geq x_{1}(t)$ and $A$ is a monotonous operator.

Theorem 3.3. Assume that $H_{4}, H_{5}$ and $H_{6}$ hold, and the mapping $A: A v_{n}=$ $v_{n+1}, A w_{n}=w_{n+1}$ are defined as:

$$
\begin{gathered}
D^{q} v_{n+1}=f\left(t, v_{n}\right)-M\left(v_{n+1}(t)-v_{n}(t)\right), \quad v_{n+1}(0)=-\int_{0}^{T} w_{n}(s) d s+d, \\
D^{q} w_{n+1}=f\left(t, w_{n}\right)-M\left(w_{n+1}(t)-w_{n}(t)\right), \quad w_{n+1}(0)=-\int_{0}^{T} v_{n}(s) d s+d .
\end{gathered}
$$

Then there exist monotone sequences $\left\{v_{n}\right\},\left\{w_{n}\right\}$ which converges to the same function $\phi(t)$ which is the unique solution of problem (1.1) with $\lambda=-1$.

Proof. The definition of $A$ gives $A v_{n-1}=v_{n}, A w_{n-1}=w_{n}$. From (a) and (b) in Lemma 3.2 there is no difficulty to get: for $t \in J, v_{0} \leq v_{1} \leq \ldots \leq v_{n-1} \leq$ $v_{n} \leq w_{n} \leq w_{n-1} \leq \ldots \leq w_{2} \leq w_{1} \leq w_{0}$. Apparently, there exist $v, w$ such that $\lim _{n \rightarrow \infty} v_{n}(t)=v(t), \lim _{n \rightarrow \infty} \overline{w_{n}}(t)=w(t)$. And $v, w$ are solutions to (1.1).

Setting $p=w-v$, we have

$$
\begin{aligned}
D^{q} p & =f(t, w(t))-f(t, v(t)) \\
& \leq M[w(t)-v(t)] \\
& =p(t) \\
p(0) & =w(0)-v(0) \\
& =\lim _{n \rightarrow \infty}\left[w_{n}(0)-v_{n}(0)\right] \\
& =\lim _{n \rightarrow \infty}\left\|w_{n-1}-v_{n-1}\right\| \\
& =0 .
\end{aligned}
$$

With Lemma 2.3 again, we get $p(t) \leq 0$, which means $v(t) \geq w(t)$. Considering $v(t) \leq w(t)$, we have $v(t)=w(t):=\phi(t)$. We have got the uniqueness of solution to (1.1) with $\lambda=-1$. The proof of Theorem 3.3 is complete.

Acknowledgements: The second author was supported by the National Natural Science Foundation of China (No 10701023), and in part by E-Institutes of Shanghai Municipal Education Commission (No N.E03004). 


\section{REFERENCES}

1. J.H. He, Nonlinear oscillation with fractional derivative and its applications, International Conference on Vibrating Engineering, 288-291, Dalian, China, 1998. 1

2. J.H. He, Some applications of nonlinear fractional differential equations and their approximations, Bull. Sci. Technol. 15 (1999), 86-90. 1

3. J.H. He, Approximate analytical solution for seepage flow with fractional derivatives in porous media, Computer Methods in Applied Mechanics and Engineering 167 (1998), 5768. 1

4. J.H. He and X.H. Wu, Variational iteration method: New development and applications, Computers \& Mathematics with Applications 54 (2007), 881-894. 1

5. T.Jankwoski, Differential equations with integral boundary conditions, Journal of Computational and Applied Mathematic 147 (2002), 1-8. 1

6. V.Lakshmikantham and A.S.Vatsala, Basic theory of fractional differential equation, Nonlinear Anal. TMA, doi:10.1016/j.na.2007.08.042. 1

7. V.Lakshmikantham and A.S.Vatsala, General uniqueness and monotone iterative technique for fractional differential equations, Appl. Math. Lett., doi:10.1016/j.aml.2007.09.006. 1

8. V.Lakshmikantham and A.S.Vatsala, Theory of fractional differential inequalities and applications, Communications in Applied Analysis, (in press). 1, 2

9. S. Momani and Z. Odibat, Numerical comparison of methods for solving linear differential equations of fractional order, Chaos Solitons \& Fractals 31 (2007), 1248-1255. 1

10. Z. Odibat and S. Momani, Modified homotopy perturbation method: Application to quadratic Riccati differential equation of fractional order, Chaos Solitons \& Fractals 36 (2008), 167174. 1

11. Z. Odibat and S. Momani, Application of variational iteration method to nonlinear differential equations of fractional order, International Journal of Nonlinear Sciences and Numerical Simulation 7 (2006), 27-34. 1

12. Z. Odibat, Solitary solutions for the nonlinear dispersive $K(m, n)$ equations with fractional time derivatives, Physics Letters A 370 (2007), 295-301. 1

13. I.Podlubny, Fractional Differential Equations, Academic Press, San Diego, 1999. 1

14. S.G. Samko, A.A. Kilbas and O.I. Marichev, Fractional Integrals and Derivatives: Theory and Applications, Gordon and Breach, New York, 1993. 1

15. Q. Wang, Homotopy perturbation method for fractional KdV-Burgers, Chaos Solitons \& Fractals 35 (2008), 843-850. 1

${ }^{1}$ Department of Applied Mathematics, Donghua University, Shanghai 201620, ChINA.

E-mail address: tigerwtg@hotmail.com

2 Department of Applied Mathematics, Donghua University, Shanghai 201620, China.

E-mail address: fxie@dhu.edu.cn 